\title{
The Impact of the One-Day Clinic Diabetes Mellitus Management Model on Perinatal Outcomes in Patients with Gestational Diabetes Mellitus
}

\author{
Yan-Min Cao* \\ Wei Wang* \\ $\mathrm{Na}-\mathrm{Na} \mathrm{Cai}$ \\ Min Ma \\ Jie Liu \\ Peng Zhang \\ Lian Xue \\ Yuan-Yuan Liu
}

Department of Internal Medicine, The Fourth Hospital of Shijiazhuang (Maternal Hospital Affiliated to Hebei Medical University), Shijiazhuang, 050000,

People's Republic of China

*These authors contributed equally to this work
Correspondence: $\mathrm{Na}-\mathrm{Na} \mathrm{Cai}$; Min Ma Department of Internal Medicine, The Fourth Hospital of Shijiazhuang (Maternal Hospital Affiliated to Hebei Medical University), No. 16, Tangu North Street, Shijiazhuang, 050000, People's Republic of China

Tel +86031189911662

Fax +8603 II 899II 669

Email cainanamed@I63.com;

mamin_drma@I63.com
Objective: The aim of the present study was to explore the impact of the one-day clinic diabetes mellitus (DM) management model on perinatal outcomes in patients with gestational diabetes mellitus (GDM).

Methods: A total of 995 patients who underwent prenatal checkups at our hospital and were diagnosed with GDM by oral glucose tolerance test (OGTT) screening at 24-28 weeks of gestational age were enrolled between December 2018 and August 2020. The patients were randomly divided into a study group (541 cases) and a control group (454 cases). One-day clinic intervention for DM was conducted in the study group, while individualized dietary interventions and exercise instruction were given in the control group. The perinatal outcomes of patients were compared between the two groups.

Results: In the study group, maternal weight gain, fasting blood glucose before delivery, the incidence of abnormality in postpartum OGTT, and abnormality of pancreatic islet function were lower than in the control group, and the differences were statistically significant $(P<$ $0.05)$. The percentage of patients with insulin treatment in the study group was higher than in the control group, and the difference was statistically significant $(P<0.05)$. The incidence of premature rupture of membranes, macrosomia, and neonatal jaundice was lower in the study group than in the control group, and the differences were statistically significant $(P<0.05)$. Conclusion: The one-day clinic DM management model can effectively control weight gain and blood glucose levels during pregnancy in patients with GDM, resulting in a higher recovery rate of blood glucose and islet function after delivery and a lower incidence of premature rupture of membranes, macrosomia, and neonatal jaundice. The one-day clinic DM management model could therefore have profound implications for reducing and delaying the onset of postpartum type 2 diabetes in patients with GDM.

Keywords: gestational diabetes, one-day clinic, blood glucose, body weight, perinatal outcome

\section{Introduction}

Gestational diabetes mellitus (GDM) is defined as diabetes mellitus (DM) first detected after pregnancy. With the improvement of living standards, the incidence of GDM has been gradually increasing, and the International Diabetes Federation (IDF) found that the global prevalence in 2017 of patients in a combined hyperglycemic state during pregnancy was $16.2 \%$, of which GDM accounted for $86.4 \%{ }^{1}$ Moreover, GDM has 
been found to increase the risk of adverse perinatal outcomes, ${ }^{2}$ such as hypertension during pregnancy, amniotic fluid abnormalities, fetal malformations, macrosomia, neonatal respiratory distress syndrome, and neonatal hypoglycemia. In addition, the long-term maternal risk of type 2 diabetes mellitus (T2DM), obesity, hypertension, metabolic syndrome, and atherosclerosis is significantly higher following GDM. Therefore, the proper management of hyperglycemia during pregnancy is an important opportunity to reduce the risk of these conditions. ${ }^{3,4}$

Most patients with GDM can control their blood glucose within the normal range through standardized management, diet adjustment, reasonable exercise, and timely monitoring. Therefore, on the basis of individualized dietary interventions and exercise guidance, patients were advised to participate in the one-day clinic DM management model in our hospital. They achieved good results, which are reported in this paper.

\section{Materials and Methods General Data}

A total of 995 pregnant women with GDM who underwent prenatal checkups at our hospital were enrolled between December 2018 and August 2020. These patients were randomly divided into a study group (541 patients) and a control group (454 patients). At enrollment, the average age of the study group was $29.87 \pm 3.77$ years, and the average gestational age was $26.74 \pm 2.66$ weeks. The average age of the control group at enrollment was $29.98 \pm 4.08$ years, and the average gestational age was $26.31 \pm 4.29$ weeks. There were no statistically significant differences between the two groups in general characteristics, including age, height, prepregnancy weight, pre-pregnancy body mass index (BMI), family history of DM, enrollment weight, enrollment BMI, and gestational age at enrollment $(P>0.05)$, indicating that the data were comparable. This study was conducted in accordance with the Declaration of Helsinki (as was revised in 2013) and approved by the Ethics Committee of The Fourth Hospital of Shijiazhuang. Written informed consent was obtained from all participants.

\section{Inclusion and Exclusion Criteria}

According to the guidelines published by the American Diabetes Association in 2011, DM combined with pregnancy is diagnosed when the fasting blood glucose (FBG) $\geq 7.0$ $\mathrm{mmol} / \mathrm{L}$, glycated hemoglobin $(\mathrm{HbA} 1 \mathrm{C}) \geq 6.5 \%$, or random blood glucose $(\mathrm{BG}) \geq 11.1 \mathrm{mmol} / \mathrm{L}$ at the first pregnancy checkup with the presence of symptoms. In addition to the above mentioned DM combined with pregnancy women, pregnant women at a gestational age of 24-28 weeks were selected to take a $75 \mathrm{~g}$ oral glucose tolerance test (OGTT) to detect BG at three time points: FBG, $1 \mathrm{~h} \mathrm{BG}$ and $2 \mathrm{~h}$ BG. The cut-off points were FBG of $5.1 \mathrm{mmol} / \mathrm{L}, 1 \mathrm{~h} \mathrm{BG}$ of $10 \mathrm{mmol} /$ $\mathrm{L}$, and $2 \mathrm{~h} \mathrm{BG}$ of $8.5 \mathrm{mmol} / \mathrm{L}$, and patients with a $\mathrm{BG}$ equal to or higher than these values were diagnosed with GDM.

\section{Methods}

In the control group, individualized dietary interventions and exercise instruction were conducted, and patients were instructed to review regularly. In the study group, individualized dietary interventions, exercise instruction, and participation in the one-day clinic for GDM were conducted.

The one-day clinic was attended by an endocrinologist and a nurse, with the measurement of FBG and fetal heartbeat detected from 7:15 to 7:30 a.m., a meal suitable for diabetic patients provided at 7:50 a.m., and a gymnastics session suitable for pregnant women given at 8:50 a.m. A lecture was delivered at 9:40 a.m., which included information about making nutritious meals, the diagnosis and hazards of GDM, susceptibility factors, dietary control methods, food exchange, exercise, BG monitoring and review, postpartum review, and precautions; psychological guidance was also provided. Each patient's two-hour postprandial BG (2 h PBG) was detected at 9:50 a.m., followed by a rest. BG was detected again at 12:00 p.m., followed by another meal and a rest. Another gymnastics session was conducted at 1:00 p.m., followed by a video presentation about childbirth and breastfeeding. $2 \mathrm{~h}$ PBG was detected again at 2:00 p.m., followed by individualized guidance based on the detected BG, a question-and-answer session, post-session quiz, questionnaire distribution, and enrollment in a diabetes management WeChat group. A third meal was provided at 3:30 p.m., and the clinic activities were completed at 4:00 p.m. After the clinic, the WeChat group regularly released information related to DM, provided follow-up answers, and supervised the patients in order to review their condition. The patients were also followed up with timely one-to-one guidance.

\section{Observation Indicators}

Prenatal, enrolment and pre-delivery body weight and BMI were recorded respectively in both groups, in addition, FBG and $\mathrm{HbA} 1 \mathrm{C}$ were recorded at enrolment, before delivery and postpartum. Insulin application, gestational age at delivery, mode of delivery, and the number of cases with gestational hypertension, excessive amniotic fluid, 
premature rupture of membranes, placental abruption, macrosomia, premature birth, neonatal hypoglycemia, neonatal jaundice, and neonatal respiratory distress syndrome were also recorded in detail.

Moreover, OGTT and insulin release test results were performed postpartum. The insulin release test was used to evaluate the function of maternal islet cells.

Normal value of OGTT: Fasting blood glucose ranged from 3.9 to $6.1 \mathrm{mmol} / \mathrm{L}$. $0.5 \sim 1$ hour after oral administration of glucose, blood glucose reached a peak (generally $7.8 \sim$ $9.0 \mathrm{mmol} / \mathrm{L}$ ), with a peak value of less than $11.1 \mathrm{mmol} / \mathrm{L}$. After 2 hours of oral administration of glucose, the blood glucose was less than $7.8 \mathrm{mmol} / \mathrm{L}$, and the blood glucose returned to the fasting level after 3 hours. Test results outside the normal range are considered abnormal OGTT results.

Insulin release test results: After 8-12 hours of fasting, about $250 \sim 300 \mathrm{~mL}$ of $75 \mathrm{~g}$ anhydrous glucose solution was given to the puerpera within 5 minutes. Plasma insulin levels were measured at fasting and $0.5 \mathrm{~h}, 1 \mathrm{~h}, 2 \mathrm{~h}, 3 \mathrm{~h}$ after glucose administration.

Normal value of insulin release test: basal plasma insulin in normal subjects was $5 \sim 20 \mathrm{mU} / \mathrm{L}$, and it rose to the peak value after $30 \sim 60 \mathrm{~min}$ of oral glucose (it could be $5 \sim 10$ times of the basal value, and most of them were $50 \sim 100$ $\mathrm{mU} / \mathrm{L})$. Three hours after oral glucose the insulin was reduced to the baseline level. Test results outside the normal range are considered to be abnormal islet function.

\section{Statistical Analysis}

SPSS 23.0 software was used for statistical analysis. The measurement data were expressed as $\bar{x} \pm \mathrm{s}$ and a $t$-test was used for comparison between the two groups. The countable data were expressed as percentages $(\%)$ and the $\chi^{2}$ test was adopted for comparison between the two groups. $P<0.05$ was considered statistically significant.

\section{Results \\ General Characteristics}

As shown in Table 1, there were no statistically significant differences in age, height, pre-pregnancy weight, prepregnancy BMI, family history of DM, enrollment weight, enrollment BMI, and gestational age at enrollment between the two groups $(P>0.05)$.

\section{Changes in the Body Weight (BW) in the Pregnant Women with GDM After Intervention Therapy}

There were no statistically significant differences between the two groups in weight gain and BMI growth from prepregnancy to enrollment $(P>0.05)$. But the weight gain and BMI growth of the women in the study group were significantly lower than those in the control group from enrollment to pre-delivery and during pregnancy $(P<$ 0.05). (See Table 2)

\section{Changes in BG After Intervention Therapy}

There were no statistically significant differences in FBG and $\mathrm{HbA} 1 \mathrm{C}$ between the two groups at enrollment $(P>0.05)$. In the study group, FBG and $\mathrm{HbA} 1 \mathrm{C}$ were lower before delivery than at enrollment $(P<0.05)$. But in the control group, in the same time period FBG was lower and $\mathrm{HbA} 1 \mathrm{C}$ was higher $(P<0.05)$. Before delivery, both FBG and $\mathrm{HbA} 1 \mathrm{C}$ in the study group were numerically lower than those in the control group, but only the difference in FBG was statistically significant $(P<0.01)$, while the difference in $\mathrm{HbA1C}$ did not

Table I Comparison of the General Characteristics Between the Two Groups ( $\bar{x} \pm s)$

\begin{tabular}{|l|c|c|c|}
\hline & Study Group & Control Group & P \\
\hline $\mathbf{n}$ & $\mathbf{5 4 I}$ & $\mathbf{4 5 4}$ & 0.712 \\
Age (year) & $29.87 \pm 3.77$ & $29.98 \pm 4.08$ & 0.180 \\
Height (cm) & $161.43 \pm 5.37$ & $161.87 \pm 4.70$ & 0.167 \\
Pre-pregnancy weight (Kg) & $62.27 \pm I 1.73$ & $62.27 \pm 10.97$ & 0.059 \\
Pre-pregnancy BMI & $24.30 \pm 4.86$ & $23.76 \pm 4.04$ & 0.194 \\
Family history of DM (\%) & $194 / 54 I(35.9)$ & $181 / 454(39.9)$ & 0.307 \\
Enrollment weight (Kg) & $70.20 \pm 12.01$ & $71.45 \pm 11.00$ & 0.104 \\
Enrollment BMI & $27.72 \pm 4.87$ & $27.25 \pm 4.02$ & 0.052 \\
Gestational age at enrollment & $26.74 \pm 2.66$ & $26.31 \pm 4.29$ & \\
\hline
\end{tabular}

Abbreviations: BMI, body mass index; DM, diabetes mellitus. 
Table 2 Comparison of the Changes in the Body Weight Between the Two Groups $(\bar{x} \pm \mathrm{s})$

\begin{tabular}{|l|c|c|c|c|}
\hline & & Study Group & Control Group & $P$ \\
\hline Weight gain $(\mathrm{Kg})$ & From pre-pregnancy to the enrollment & $8.92 \pm 5.84$ & $9.15 \pm 5.14$ & $0.5 \mathrm{I}$ \\
& From enrollment to pre-delivery & $2.61 \pm 4.20$ & $1.48 \pm 5.23$ & $<0.01$ \\
& Throughout pregnancy & $11.53 \pm 5.92$ & $13.70 \pm 6.71$ & $<0.01$ \\
\hline \multirow{2}{*}{ BMI growth $\left(\mathrm{Kg} / \mathrm{m}^{2}\right)$} & From pre-pregnancy to the enrollment & $3.42 \pm 2.19$ & $3.29 \pm 1.94$ & 0.592 \\
& From enrollment to pre-delivery & $1.00 \pm 1.61$ & $1.70 \pm 1.98$ & $<.01$ \\
& Throughout pregnancy & $4.42 \pm 2.23$ & $5.22 \pm 2.54$ & $<0.01$ \\
\hline
\end{tabular}

Abbreviation: BMI, body mass index.

meet the statistical standard. But there was significant differences in the change of FBG and $\mathrm{HbAlC}$ between the two groups from enrollment to pre-delivery $(P<0.01)$. Moreover, The therapeutic rate of insulin in the study group was higher than which in the control group $(P<0.05)$. (See Table 3)

\section{Postpartum BG and Islet Function}

The postpartum review rate and the differences in postpartum FBG and $\mathrm{HbA1C}$ were not statistically significant between the two groups $(P>0.05)$. The proportion of abnormal postpartum OGTT results and postpartum islet function in study group was lower than which in control group $(P<0.05)$. The reexamination rate was $61.3 \%$ in the study group but only $21.4 \%$ in the control group, and the difference between the two groups was statistically significant $(P<0.01)$. (See Table 4)

\section{Perinatal Complications and Modes of Delivery}

The incidence of premature rupture of membranes was lower in the study group than in the control group, and the difference was statistically significant $(P<0.05)$. There were no statistically significant differences between the two groups in terms of the incidence of perinatal complications, gestational hypertension, preeclampsia, excessive/low amniotic fluid, placental abruption, and mode of delivery $(P>0.05)$. (See Table 5)

\section{Outcomes of Neonates}

The incidence of macrosomia and neonatal jaundice in the study group was lower than in the control group, and the difference was statistically significant $(P<0.05)$. The differences between the two groups in the incidence of neonatal complications, gestational age of newborn, prematurity, neonatal hypoglycemia, neonatal respiratory distress syndrome, intrauterine fetal death, and fetal distress were not statistically significant $(P>0.05)$. (See Table 6)

\section{Discussion}

With the continuous improvement of living standards and the liberalization of the two-child policy in China, the incidence of GDM is increasing every year, seriously affecting the

Table 3 Comparison of the Changes in Blood Glucose Between the Two Groups ( $\bar{x} \pm \mathrm{s})$

\begin{tabular}{|c|c|c|c|c|}
\hline & & Study Group & Control Group & $\mathbf{P}$ \\
\hline \multirow[t]{4}{*}{ Fasting blood glucose $(\mathrm{mmol} / \mathrm{l})$} & At enrollment & $5.56 \pm 1.00$ & $5.49 \pm 0.86$ & 0.246 \\
\hline & Before delivery & $4.82 \pm 0.57$ & $4.98 \pm 0.55$ & $<0.01$ \\
\hline & Difference & $0.74 \pm 0.96$ & $0.5 I \pm 0.96$ & $<0.01$ \\
\hline & Po & 0.000 & 0.000 & \\
\hline \multirow[t]{4}{*}{ HbAIC (\%) } & At enrollment & $5.39 \pm 0.52$ & $5.32 \pm 0.57$ & 0.067 \\
\hline & Before delivery & $5.33 \pm 0.44$ & $5.39 \pm 0.46$ & 0.059 \\
\hline & Difference & $0.05 \pm 0.53$ & $-0.07 \pm 0.61$ & $<0.01$ \\
\hline & Po & 0.023 & 0.023 & \\
\hline \multicolumn{2}{|l|}{ Rate of insulin medication (\%) } & $39 / 541(7.2)$ & $17 / 454(3.7)$ & 0.018 \\
\hline \multirow[t]{3}{*}{ Condition of insulin medication [n (\%)] } & Aspart+Detemir & $27 / 54 \mid(5.0)$ & I5/454 (3.3) & 0.187 \\
\hline & Aspart & $2 / 541(0.4)$ & $\mathrm{I} / 454(0.2)$ & 0.667 \\
\hline & Detemir & $10 / 54 \mid(1.8)$ & $\mathrm{I} / 454(0.2)$ & 0.014 \\
\hline
\end{tabular}


Table 4 Comparison of Postpartum Blood Glucose and Islet Function Between the Two Groups $(\bar{x} \pm s)$

\begin{tabular}{|l|c|c|c|}
\hline & Study Group & Control Group & $P$ \\
\hline Postpartum FBG & $5.01 \pm 0.52$ & $5.00 \pm 0.60$ & 0.826 \\
Postpartum HbAIC & $5.46 \pm 0.39$ & $5.45 \pm 0.5 \mathrm{I}$ & 0.928 \\
Postpartum review rate I (\%) & $436 / 541(80.6)$ & $355 / 454(78.2)$ & $0.35 \mathrm{I}$ \\
Proportion of abnormal postpartum OGTT results (\%) & $42 / 332(12.3)$ & $21 / 97(21.6)$ & 0.028 \\
Proportion of abnormal postpartum islet function (\%) & $102 / 332(30.7)$ & $42 / 97(43.3)$ & 0.021 \\
Postpartum review rate 2 (\%) & $332 / 541(61.3)$ & $97 / 454(21.4)$ & $<0.01$ \\
\hline
\end{tabular}

Abbreviation: FBG, fasting blood glucose.

Table 5 Comparison of Perinatal Complications and Modes of Delivery Between the Two Groups $\left(\chi^{2}\right)$

\begin{tabular}{|c|c|c|c|}
\hline & Study Group & Control Group & $\mathbf{P}$ \\
\hline Perinatal maternal complications (\%) & $97 / 54 \mid(17.9)$ & $96 / 454(21.1)$ & 0.201 \\
\hline Gestational hypertension (\%) & $|7 / 54|(3.1)$ & 13/454 (2.9) & 0.798 \\
\hline Pre-eclampsia (severe) (\%) & $26 / 54 I(4.8)$ & $13 / 454(2.9)$ & 0.116 \\
\hline Premature rupture of membranes (\%) & $36 / 541(6.7)$ & $53 / 454(11.7)$ & 0.006 \\
\hline Excessive/low amniotic fluid (\%) & $16 / 54 \mid(3.0)$ & $10 / 454(2.2)$ & 0.457 \\
\hline Placental abruption (\%) & $3 / 54 \mid(0.6)$ & $3 / 454(0.7)$ & 0.829 \\
\hline Cesarean section (\%) & $248 / 54 I(45.8)$ & 195/454 (43.0) & 0.361 \\
\hline Obstetric forceps (\%) & $6 / 54 I(1.1)$ & $10 / 454(2.2)$ & 0.172 \\
\hline Induction of labor (\%) & $0 / 54 \mid(0.0)$ & $3 / 454(0.7)$ & 0.058 \\
\hline
\end{tabular}

Table 6 Comparison of Neonatal Outcomes Between the Two Groups $\left(\chi^{2}\right)$

\begin{tabular}{|c|c|c|c|}
\hline & Study Group & Control Group & $\mathbf{P}$ \\
\hline Gestational age of the neonate & $38.54 \pm 1.38$ & $38.52 \pm 1.98$ & 0.815 \\
\hline Neonatal complication (\%) & $90 / 54 \mid(16.6)$ & $94 / 454(20.7)$ & 0.100 \\
\hline Macrosomia (\%) & $44 / 541(8.1)$ & $69 / 454(15.2)$ & $<0.01$ \\
\hline Premature infant (\%) & $46 / 541(8.5)$ & $37 / 454(8.1)$ & 0.841 \\
\hline Neonatal hypoglycemia (\%) & $10 / 541(1.8)$ & $8 / 454(1.8)$ & 0.919 \\
\hline Neonatal jaundice (\%) & $72 / 54 \mid(13.3)$ & $81 / 454(17.9)$ & 0.047 \\
\hline Neonatal respiratory distress syndrome (\%) & $0 / 54 I(0.0)$ & $1 / 454(0.2)$ & 0.275 \\
\hline Fetal death in the uterus (\%) & $0 / 54 I(0.0)$ & $2 / 454(0.4)$ & 0.122 \\
\hline Fetal distress (\%) & $7 / 54 \mid(1.3)$ & $7 / 454(1.5)$ & 0.741 \\
\hline
\end{tabular}

health and prognosis of mothers and neonates. The effective control of BG in pregnant women with GDM and the avoidance of complications is therefore of great significance to maternal and infant health and prognosis.

In the one-day clinic DM management model, unlike traditional GDM management methods, education consisting of a combination of lectures, demonstrations, and practical experience is adopted to teach patients about the use of food exchange, dietary modifications, selfmonitoring methods, precautions for $\mathrm{BG}$, and exercise. After a day of eating meals in the clinic, patients have a greater understanding of food combinations and the quantity of food in standard meals with fixed dietary energy; they also learn about suitable exercise levels and methods and the harm GDM can present to mothers and babies, allowing them to learn effective self-management methods. Patients who attend the clinic are also able to monitor the changes in their condition according to a doctor's instructions and actively follow instructions for diet control and exercise to effectively control BG and weight. This improves pregnancy outcomes and the safety of both the mother and neonate. 
Weight gain is an important control indicator for patients with GDM. It has been found that weight gain during pregnancy is an independent risk factor for fetal overgrowth in patients with GDM: excessive weight gain during pregnancy increases the neonatal birth weight by nearly $0.5 \mathrm{~kg}$, and the incidence of perinatal complications and neonatal hypoglycemia increases significantly. ${ }^{5}$ Excessive gestational weight gain in pregnant women with GDM also significantly increases the risk of developing gestational hypertension. ${ }^{6}$ However, it is important to note that inadequate weight gain in women during pregnancy also increases the risk of preterm delivery and leads to fetuses being small for their gestational age. ${ }^{7}$ Therefore, controlling weight gain during pregnancy is of great significance to patients with GDM. In the present study, the patients in the study group were asked during the one-day clinic to monitor their weight changes every week and ensure the increases stayed within a certain range. The results showed that weight gain and BMI growth in the study group between enrollment and predelivery and throughout pregnancy were lower than in the control group, indicating that the weight control of patients with GDM who underwent a unified one-day clinic for DM management was more effective. Controlling weight gain during pregnancy and ensuring it remained within the standard range also ensured a supply of nutrition to the mother and neonate and improved BG control, which is conducive to postpartum recovery.

Increased BG is a significant risk factor that increases the adverse outcomes of mothers and neonates. Previous studies have shown that GDM is closely correlated with the occurrence of hypertension during pregnancy ${ }^{8}$ and that poor $\mathrm{BG}$ control can lead to an increased incidence of long-term suffering for both the mother and infant, including T2DM, obesity, hypertension, metabolic syndrome, abnormal cardiovascular metabolism, and other conditions. ${ }^{9-11}$ Although hyperglycemia during pregnancy generally disappears after delivery, prolonged insulin resistance and $\beta$-cell dysfunction are also observed in patients with GDM and can persist after delivery. ${ }^{12}$ The present study found that the FBG and HbA1C in study groups were lower before delivery than at enrollment, and before delivery FBG in the study group was lower than that in the control group. It indicates that the patients had better glycemic control after the one-day clinic. During the postpartum review, patients in the study group had lower proportion of abnormal OGTT results and abnormal pancreatic islet function than those in the control group, which suggests that, after adopting the therapeutic model of the one- day clinic, patients will have better glycemic control and a higher recovery rate of postpartum BG and islet function; this could have profound significance for reducing and delaying the occurrence of postpartum T2DM in patients with GDM. ${ }^{13}$ However, this result needs to be further confirmed, as patients in the control group had poor compliance and the review rates of OGTT and the insulin release test were lower in the control group than in the study group.

In the present study, there was a significant difference between the two groups in the rate of insulin medication, with the study group having a higher rate of insulin medication than the control group; this might be due to the greater compliance and higher regular review rate of the patients who attended the one-day clinic for DM management. During the study, insulin therapy was given to patients in accordance with the standard of their BG control and in a timely manner; if a patient only had high FBG, small doses of the Insulin Detemir were given to control BG. The control group, however, had poor compliance and low regular review rates, with many patients not receiving insulin therapy in a timely manner when their BG was poorly controlled. The results of the present study suggest that, for patients with GDM whose BG control is adequate after diet control and reasonable exercise, the timely application of insulin therapy is beneficial for BG control and the recovery of postpartum islet function and reduces the risk of adverse pregnancy outcomes, which is consistent with the results of previous studies. ${ }^{14}$

Conventional individualized dietary interventions and exercise instruction are limited by time and the comprehension of the patient and are often formal and lacking in operability, making it difficult for pregnant women to implement the knowledge they have acquired, thus reducing compliance with the treatment. ${ }^{15}$ The one-day clinic DM management model addresses these limitations by delivering information about GDM, offering guidance on cooking meals that are suitable for people with diabetes, demonstrating suitable exercises, and establishing a WeChat management group to improve patient participation and compliance. This has resulted in achieving the goal of controlling $\mathrm{BG}$ and reducing the incidence of adverse pregnancy outcomes.

Between 15 and $45 \%$ of pregnancies in women with GDM result in macrosomia. ${ }^{16}$ Previous studies have shown that the occurrence of GDM macrosomia is correlated with the family history of diabetes in pregnant women, BMI before pregnancy, weight gain during pregnancy and fasting blood glucose increase. Among the adverse outcomes of GDM macrosomia, the risk of neonatal hypoglycemia was 
1. 599 times that of non-macrosomia. ${ }^{17}$ The developing fetus has limited ability to synthesize glucose on its own. Glucose from the mother's blood can cross the placenta, while insulin does not. Therefore, maternal high blood sugar can lead to neonatal obesity. ${ }^{18}$ Excessive nutrient storage can lead to birth weight gain or macrosomia. Most of the obesity is concentrated in the fetal abdomen and shoulders, which increases the risk of shoulder dystocia and birth injury. ${ }^{19}$ The presence of GDM, along with other risk factors such as high blood pressure and obesity during pregnancy, can also lead to premature labor and delivery. ${ }^{20}$ Therefore, the most direct adverse pregnancy outcome of GDM patients with poor blood glucose and weight control is macrosomia, which will lead to a series of other adverse pregnancy outcomes. One study found that up to 12 hours of comprehensive nutritional care significantly reduces the incidence of macrosomia in patients with GDM. ${ }^{21}$ It has also been shown that dietary control and appropriate exercise can effectively control BG and reduce the incidence of adverse pregnancy outcomes in patients with GDM. ${ }^{22}$ The present study aimed to investigate the impact on perinatal outcomes in patients with GDM of the one-day clinic DM management model compared with individualized dietary intervention and exercise guidance. The study found that the incidence of premature rupture of membranes, macrosomia, and neonatal jaundice were lower in the study group than in the control group, but there were no statistically significant differences in the incidence of perinatal complications, gestational hypertension, preeclampsia, excessive/low amniotic fluid, placental abruption, mode of delivery, neonatal complications, preterm birth, neonatal hypoglycemia, neonatal respiratory distress syndrome, intrauterine fetal death, and fetal distress between the two groups. Patients included in the one-day clinic DM management had good compliance. They had better BG and weight control, which reduced the incidence of macrosomia. However, due to the complex and varied causes of adverse pregnancy outcomes, blood glucose and body weight were only part of them. In this study, the incidence of premature rupture of membranes, macrosomia and neonatal jaundice were different, while no difference was found in other adverse pregnancy outcomes.

The development of GDM is related to the impaired glucose tolerance caused by the dysfunction of maternal pancreatic $\beta$-cells. ${ }^{23}$ Glucose passes through the placenta to the fetus and causes increased fetal insulin secretion, stimulating fetal growth, resulting in macrosomia and Older than gestational age (LGA). ${ }^{24}$ In addition, during normal pregnancies, the mother develops progressive insulin resistance under the influence of placental hormones. In order to maintain glucose homeostasis in the body under these conditions, pancreatic $\beta$-cells must release more insulin than usual to regulate maternal blood sugar levels and ensure that there is enough nutrients to support the healthy growth of the fetus. However, GDM occurs when maternal pancreatic $\beta$-cells are unable to adapt to the metabolic changes that accompany pregnancy. In this study, on the basis of individualized diet intervention and exercise guidance, pregnant women in the study group were also given one-day clinic DM management intervention. Through explaining GDM related knowledge, displaying diabetic diet, demonstrating exercise, establishing WeChat management group, timely oneto-one guidance was provided to patients until postpartum. This management approach improves patient participation and compliance, enables patients to follow a standardized diet and exercise regimen for GDM in the long term, and enables patients with poor glycemic control to initiate insulin therapy in a timely manner. Good control of BG during pregnancy can reduce insulin resistance, reduce the occurrence of macrosomia, protect the function of maternal pancreatic $\beta$-cell, and thus play a good role in the recovery of postpartum BG and pancreatic islet function.

\section{Conclusion}

The adoption of the one-day clinic DM management model to follow up patients with GDM and provide timely one-to-one guidance can effectively control weight gain and BG during pregnancy, resulting in higher rates of postpartum recovery of $\mathrm{BG}$ and islet function and reducing the incidence of premature rupture of membranes, macrosomia, and neonatal jaundice. However, the incidence of gestational hypertension, preeclampsia, excessive/low amniotic fluid, placental abruption, mode of delivery, premature birth, neonatal hypoglycemia, neonatal respiratory distress syndrome, intrauterine fetal death, and fetal distress in patients who receive intervention with the one-day clinic model does not differ significantly from the incidence of these conditions in patients who receive conventional individualized dietary intervention and exercise instruction. The one-day clinic DM management model could have profound implications for reducing and delaying the onset of postpartum T2DM in patients with GDM.

\section{Disclosure}

The authors declare that they have no competing interests. 


\section{References}

1. International Diabetes Federation. IDF Diabetes Atlas. 8th ed. Brussels: International Diabetes Federation; 2017.

2. Metzger BE, Buchanan TA, Coustan DR, et al. Summary and recommendations of the fifth international workshop-conference on gestational diabetes mellitus. Diabetes Care. 2007;30(Suppl 2):S251S260. doi:10.2337/dc07-s225

3. Wu LL, Niu JM. [Gestational diabetes mellitus: an opportunity for maternal and infant cardiometabolic risk prevention]. Chin J Pract Intern Med. 2018;38:527-530. [Chinese].

4. Chen LW, Soh SE, Tint MT, et al. Combined analysis of gestational diabetes and maternal weight status from pre-pregnancy through post-delivery in future development of type 2 diabetes. Sci Rep. 2021;11:5021. doi:10.1038/s41598-021-82789-x

5. Parellada CB, Asbjörnsdóttir B, Ringholm L, Damm P, Mathiesen ER. Fetal growth in relation to gestational weight gain in women with type 2 diabetes: an observational study. Diabet Med. 2014;31:1681-1689. doi:10.1111/dme.12558

6. Egan AM, Dennedy MC, Al-Ramli W, Heerey A, Avalos G, Dunne F. ATLANTIC-DIP: excessive gestational weight gain and pregnancy outcomes in women with gestational or pregestational diabetes mellitus. J Clin Endocrinol Metab. 2014;99:212-219. doi:10.1210/jc.2013-2684

7. Goldstein RF, Abell SK, Ranasinha S, et al. Association of gestational weight gain with maternal and infant outcomes: a systematic review and meta-analysis. JAMA. 2017;317:2207-2225. doi:10.1001/ jama.2017.3635

8. Corrigan L, O'Farrell A, Moran P, Daly D. Hypertension in pregnancy: prevalence, risk factors and outcomes for women birthing in Ireland. Pregnancy Hypertens. 2021;24:1-6. doi:10.1016/j. preghy.2021.02.005

9. Mai C, Wang B, Wen J, Lin X, Niu J. Lipoprotein-associated phospholipase A2 and AGEs are associated with cardiovascular risk factors in women with history of gestational diabetes mellitus. Gynecol Endocrinol. 2014;30:241-244. doi:10.3109/09513590.2013.871522

10. Tam WH, Ma RCW, Ozaki R, et al. In utero exposure to maternal hyperglycemia increases childhood cardiometabolic risk in offspring. Diabetes Care. 2017;40:679-686. doi:10.2337/dc16-2397

11. Grunnet LG, Hansen S, Hjort L, et al. Adiposity, dysmetabolic traits, and earlier onset of female puberty in adolescent offspring of women with gestational diabetes mellitus: a clinical study within the Danish National Birth Cohort. Diabetes Care. 2017;40:1746-1755. doi:10.2337/dc17-0514

12. Miao Z, Wu H, Ren L, et al. Long-term postpartum outcomes of insulin resistance and $\beta$-cell function in women with previous gestational diabetes mellitus. Int $J$ Endocrinol. 2020;2020:7417356. doi:10.1155/2020/7417356
13. Cui JJ, Li ZY, Wang ZL. [Effects of gestational diabetes mellitus on long-term abnormal glucose metabolism in women]. Chin J Pract Gynecol Obstet. 2021;37:152-156. [Chinese].

14. Di CH. [Effects of insulin therapy on pregnancy outcome in patients with gestational diabetes mellitus]. Chin J Mod Drug Appl. 2021;15:205-207. [Chinese].

15. Sun X, Su SP, Sun WJ, Zhang L, Zhou YF, Yang HX. [Evaluation of diabetic pregnancy outcome and one-day care for gestational diabetes mellitus after application of new diagnostic criteria]. Chin J Perinat Med. 2014;17:186-190. [Chinese].

16. Kc K, Shakya S, Zhang H. Gestational diabetes mellitus and macrosomia: a literature review. Ann Nutr Metab. 2015;66(Suppl 2):14-20. doi:10.1159/000371628

17. Chen YR, Duan XK, Liu CF, et al. [Analysis of influencing factors and pregnancy outcomes of macrosomia among pregnant women with diabetes in a hospital in Zhengzhou City from 2014 to 2018]. Chin J Gen Pract. 2021;19:959-960. [Chinese].

18. Logan KM, Gale C, Hyde MJ, Santhakumaran S, Modi N. Diabetes in pregnancy and infant adiposity: systematic review and meta-analysis. Arch Dis Child Fetal Neonatal Ed. 2017;102:F65F72. doi:10.1136/archdischild-2015-309750

19. Sovio U, Murphy HR, Smith GC. Accelerated fetal growth prior to diagnosis of gestational diabetes mellitus: a prospective cohort study of nulliparous women. Diabetes Care. 2016;39:982-987. doi: $10.2337 / \mathrm{dc} 16-0160$

20. Dunn AB, Hanson L, VandeVusse L, Leslie S. Through the microbial looking glass: premature labor, preeclampsia, and gestational diabetes: a scoping review. J Perinat Neonatal Nurs. 2019;33:35-51. doi:10.1097/JPN.0000000000000375

21. Yuan K, Wang H, Chen Y, et al. A 12-hour comprehensive nutrition care benefits blood glucose level and weight gain and improves outcomes in pregnant women with gestational diabetes mellitus. Ann Palliat Med. 2020;9:661-670. doi:10.21037/apm.2020.03.16

22. Brown J, Alwan NA, West J, et al. Lifestyle interventions for the treatment of women with gestational diabetes. Cochrane Database Syst Rev. 2017;5:CD011970.

23. Plows JF, Stanley JL, Baker PN, Reynolds CM, Vickers MH. The pathophysiology of gestational diabetes mellitus. Int $J$ Mol Sci. 2018;19:3342. doi:10.3390/ijms19113342

24. Ovesen PG, Fuglsang J, Andersen MB, Wolff C, Petersen OB, David McIntyre $\mathrm{H}$. Temporal trends in gestational diabetes prevalence, treatment, and outcomes at Aarhus University Hospital, Skejby, between 2004 and 2016. J Diabetes Res. 2018;2018:5937059. doi: $10.1155 / 2018 / 5937059$

\section{Publish your work in this journal}

Diabetes, Metabolic Syndrome and Obesity: Targets and Therapy is an international, peer-reviewed open-access journal committed to the rapid publication of the latest laboratory and clinical findings in the fields of diabetes, metabolic syndrome and obesity research. Original research, review, case reports, hypothesis formation, expert opinion and commentaries are all considered for publication. The manuscript management system is completely online and includes a very quick and fair peer-review system, which is all easy to use. Visit http://www.dovepress.com/testimonials.php to read real quotes from published authors. 